\section{Ausschreibung DGNI-Pflege-Preis}

— Die Deutsche Gesellschaft für Neurolntensiv- und Notfallmedizin (DGNI) schreibt den mit 500 Euro dotierten „DGNI-Pflege-Preis" 2017 aus. Mit dem Preis sollen Pflegekräfte gewürdigt werden, die mit professionellem Wissen und Handeln zur Verbesserung der intensivmedizinischen Versorgung kritisch kranker Patienten in der Neurologie/Neurochirurgie beitragen. Einzureichen sind die Abstracts veröffentlichter oder unveröffentlichter Projektarbeiten (z.B. im Rahmen der Fachweiterbildung in Intensivpflege und Anästhesie zur Neurolntensivmedizin), die nicht älter als 24 Monate sind, bis zum 15. Oktober 2016. Eine Kommission aus fünf Neurolntensivpflegekräften und einem ärztlichen Mitglied des DGNIPräsidiums wählt drei Kandidaten aus, die ihre Arbeit als 20-MinutenVortrag auf dem Jahreskongress der DGNI in Wien präsentieren. Der Preis wird nach Bewertung von Inhalt und Darstellung vergeben.

www.dgni.de
Auszeit vom Pflegealltag

\section{Entspannt in den Urlaub und erholt zurück}

— Urlaub! Fast alle fiebern darauf hin. Die Berufsgenossenschaft für Gesundheitsdienst und Wohlfahrtspflege (BGW) gibt Tipps, damit die freien Wochen wirklich erholsam werden.

Die letzten Tage vor dem Urlaub sind für viele die schlimmsten im ganzen Jahr. Da kommt zum Packen und zur häuslichen Vorbereitung auf die Reise der berufliche Stress, aufgeschobene Arbeiten noch abzuschließen und wichtige Dinge zu übergeben - kein guter Urlaubsstart. Die Psychologin Sabine Gregersen empfiehlt, rechtzeitig realistisch einzuschätzen, was man vorher noch schafft. „Parallel startet man am besten frühzeitig mit etwaigen Reisevorbereitungen wie Packlisten oder dem Zusammenstellen der Reiseapotheke“, fügt sie hinzu. Und: „Bis zur letzten Sekunde arbeiten und dann losfahren ist genauso belastend, wie von der Urlaubsreise direkt wieder in die Arbeit einzusteigen", so Gregersen. "Mit jeweils einem Übergangstag wird alles entspannter und erholsamer."

Meistens ist ein Kontrastprogramm zum Alltag für den Urlaub genau das richtige. „Wer beispielsweise schwer körperlich arbeitet, will sich vor allem einmal ausruhen", erläutert die Psychologin. "Schon in den ersten Urlaubstagen auf Berge zu kraxeln, wäre dann purer Stress und vermutlich wenig erholsam." Beim gemeinsamen Urlaub mit Freunden sollte man sich vorher

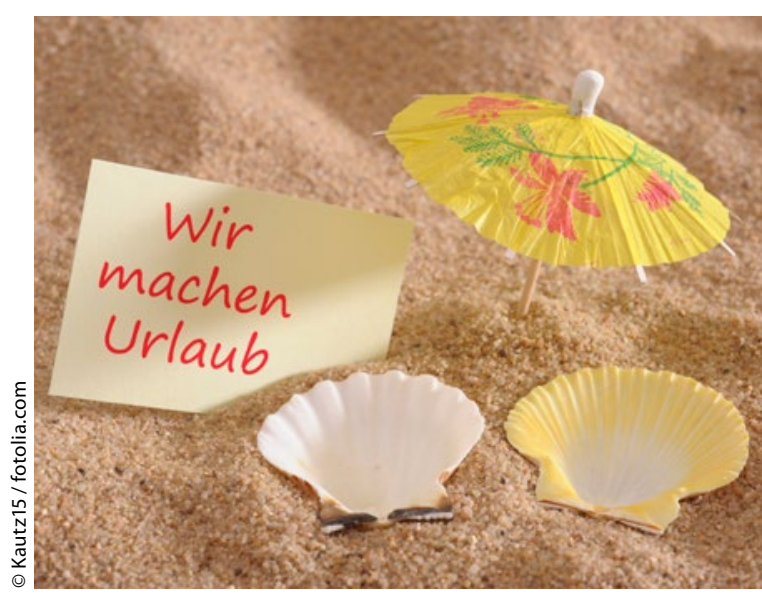

einigen, worauf Wert gelegt wird und welche Wünsche zu berücksichtigen sind. "Aber auch ein solcher Urlaub ist keine Zwangsveranstaltung", gibt Gregersen zu bedenken. „Man kann durchaus mal tageweise getrennte Wege gehen." Damit die Erholung nach dem Urlaub nicht gleich wieder dahin ist, empfiehlt sich auch für den Wiedereinstieg eine entzerrte Terminplanung. Hilfreich sind eine gute Urlaubsvertretung und - im Büro - das Einrichten eines Abwesenheitsassistenten im E-MailProgramm.

www.bgw-online.de

\title{
Digitalisierung
}

\section{Chancen und Risiken von Medizin-Apps}

- Chancen und Risiken von Fitness- und Gesundheits-Apps standen im Mittelpunkt einer Expertentagung des Bundesinstituts für Arzneimittel und Medizinprodukte (BfArM). „Bei mehr als 100.000 Gesundheits-Apps auf dem Markt wird es immer schwieriger, zwischen guten und schlechten Angeboten zu unterscheiden. Deshalb brauchen wir
Qualitäts- und Datenschutzstandards, auf die sich Bürgerinnen und Bürger verlassen können", betonte Staatssekretär Lutz Stroppe. Gesundheits-Apps müssten sowohl im privaten Bereich als auch in der Gesundheitsversorgung sicher und nutzbringend eingesetzt werden können. Aber auch App-Entwickler müssten sich orientieren. Das BfArM soll zu einer zentralen Anlaufstelle für App-Entwickler in Deutschland werden und Herstellern bei der Zulassung von "Medical Apps" beratend zur Seite stehen. Gesundheits-Apps sind ein rasant wachsender Markt.
Apps für Smartphones und Tablets geben Gesundheitstipps, helfen bei der Dosierung von Arzneimitteln und werten Fitness-Daten aus. Oft ist nicht klar, ob es sich dabei um Lifestyle-Anwendungen etwa für Fitnessdaten handelt, oder ob bereits eine medizinische Anwendung, eine "Medical App“ vorliegt. Für sie gelten die strengen Vorgaben für Medizinprodukte hinsichtlich Sicherheit, Verkehrsfähigkeit und Überwachung.

www.bfarm.de 